\title{
DYNAMICS AT INFINITY, DEGENERATE HOPF AND ZERO-HOPF BIFURCATION FOR KINGNI-JAFARI SYSTEM WITH HIDDEN ATTRACTORS
}

\author{
ZHOUCHAO WEI* \\ School of Mathematics and Physics, China University of Geosciences, Wuhan, 430074, China \\ Division of Dynamics, Technical University of Lodz, Lodz, Stefanowskiego 1/15, 90-924, Poland \\ College of Mechanical Engineering, Beijing University of Technology, Beijing, 100124, China \\ Mathematical Institute, University of Oxford, Oxford, OX2 6GG, England \\ Zhouchao.Wei@maths.ox.ac.uk,weizhouchao@163.com
}

IRENE MOROZ

Mathematical Institute, University of Oxford, Oxford, OX2 6GG, England ZHEN WANG

Department of Applied Sciences, Xijing University, Xi'an, 710123, China

JULIEN CLINTON SPROTT

Department of Physics, University of Wisconsin, Madison, WI 53706, USA

TOMASZ KAPITANIAK

Division of Dynamics, Technical University of Lodz, Lodz, Stefanowskiego 1/15, 90-924, Poland

Received (to be inserted by publisher)

\begin{abstract}
To understand the complex dynamics of Kingni-Jafari system with hidden attractors, the first objective of this paper was to study the global dynamics, and give a complete description of the dynamics of Kingni-Jafari system at infinity by using the Poincaré compactification of a polynomial vector field in $\mathbb{R}^{3}$. The second objective of this paper was to proof the existence of periodic solutions in the Kingni-Jafari system by classic Hopf bifurcation and degenerate Hopf bifurcation. Moreover, based on averaging theory, a small amplitude periodic solution that bifurcate from a zero-Hopf equilibrium was derived in the Kingni-Jafari system. The theoretical analysis and simulations demonstrate the rich dynamics of the system.
\end{abstract}

Keywords: Kingni-Jafari system; Hidden attractor; Dynamics at infinity; Zero-Hopf bifurcation; Hopf bifurcation

\section{Introduction}

In the past five decades, many 3D autonomous hyperbolic type chaotic systems have been extensively studied in science, engineering and mathematical communities. From the stability of equilibria of view,

*Author for correspondence. 
many chaotic systems have at least one unstable equilibrium, and some chaotic attractors are of Shilnikov type [Silva, 1993].

It turns out that any real dynamical system is basically nonlinear and has multiple state attractors depending on the choice of the initial conditions or system parameters. By setting these values such that it has physical meaning and the full information about every possible solution is no longer available. In the qualitative theory of differential equations, it is important to know whether a given differential system is chaotic or not. By the category of chaotic attractors either self-excited or hidden [Kuznetsov et al., 2011a, 2011b; Leonov et al., 2011, 2012; Leonov \& Kuznetsov, 2013], we call the chaotic attractors with no equilibria or only stable equilibria "hidden attractors". From a computational point of view, these hidden attractors cannot be found easily by numerical methods, but they are important and potentially problematic in engineering applications because they can lead to unexpected and potentially disastrous behavior [Kapitaniak \& Leonov, 2015].

Recently, there has been increasing attention to some unusual systems, such as those having no equilibrium [Wei, 2011; Jafari et al., 2013; Wang et al., 2012; Wei et al., 2014], stable equilibria [Yang et al., 2010; Wei \& Yang, 2011, 2012; Wang \& Chen, 2012; Molaie et al., 2013; Wei \& Zhang, 2014], a line equilibrium [Jafari \& Sprott, 2013] and coexisting attractors [Sprott et al., 2013; Li \& Sprott, 2014a, 2014b]. Motivated by the paper by Wang and Chen [Wang \& Chen, 2012], Kingni and Jafari et. al joined this exploration and performed a systematic search to find additional three-dimensional chaotic system with only one stable equilibrium [Kingni et al., 2014], which belongs to a newly introduced category of chaotic systems with hidden attractors. Nevertheless, knowledge about equilibria does not help in the localization of hidden attractors [Lao et al., 2014; Leonov et al., 2014; Jafari et al., 2015; Shahzad et al., 2015; Sharma et al.,2015a, 2015b]. Therefore, understanding the local and the global behaviors of chaotic systems with hidden attractors is of great importance [Kapitaniak \& Leonov, 2015, Wang et al., 2015; Dudkowski et al., 2015].

The present paper is a continuation of work by Kingni and Jafari et. al [Kingni et al., 2014]. It is needed to demonstrate more detailed theoretical analysis for the proposed Kingni-Jafari system with hidden attractors. The rest of this paper is organized as follows. In Section 2, the Kingni-Jafari system is introduced and stable equilibrium point can coexist with a strange attractor. In Section 3, dynamical behaviors at infinity are obtained by using the Poincaré compatification of polynomial vector field in $\mathbb{R}^{3}$. In Section 4, by using the normal form theory and symbolic computations, we present the outline of the Hopf bifurcation methods about codimension one, two and three Hopf bifurcations, in particular, how to calculate the Lyapunov coefficients related to the stability of the equilibrium. In Section 5, using averaging theory, we prove the existence of unstable periodic orbits bifurcating from the zero-Hopf equilibrium. Finally, In Section 6, we make some concluding remarks.

\section{The Kingni-Jafari system and hidden chaos}

In this section, we first give the Kingni-Jafari system proposed by Kingni et.el.[Kingni et al., 2014]:

$$
\left\{\begin{array}{l}
\dot{x}=-z, \\
\dot{y}=-x-z, \\
\dot{z}=3 x-a y+x^{2}-z^{2}-y z+b,
\end{array}\right.
$$

where the state variables $(x, y, z) \in R^{3}$ and the parameters $(a, b) \in R^{2}$. It is easy to see that when $a=0$, there is no equilibria. When $a \neq 0$, system (1) has only one equilibrium $E_{0}\left(0, \frac{b}{a}, 0\right)$. By liberalization around the $E_{0}$, the Jacobian matrix of system (1) is given by

$$
J\left(E_{0}\right)=\left(\begin{array}{ccc}
0 & 0 & -1 \\
-1 & 0 & -1 \\
3 & -a & -\frac{b}{a}
\end{array}\right) .
$$


Obviously, the following characteristic equation about the equilibrium $E_{0}$ is :

$$
\operatorname{det}\left(\lambda I-J\left(E_{0}\right)\right)=\lambda^{3}+\frac{b}{a} \lambda^{2}+(3-a) \lambda+a=0 .
$$

According to the Routh-Hurwitz criterion, the real parts of all the roots $\lambda$ are negative if and only if

$$
a>0, b>0, \frac{b}{a}(3-a)-a>0 .
$$

Therefore, $E_{0}$ is asymptotically stable if the following condition is satisfied

$$
0<a<3, b>\frac{a^{2}}{3-a} .
$$

As can be seen in Fig.1, system (1) has three types of the equilibrium $E_{0}$ in the region $\{(a, b) \mid 0<$ $a<2,0<b<3\}$. Based on the above discussions, we can find hidden chaos in above parameter regions. Therefore, in the following we focus on some behavior previously unobserved phenomenon in 3D system: hidden attractors with only one stable equilibrium, which have a basin of attraction that does not intersect with small neighborhoods of any equilibrium points. By choosing some parameter values $a=1.3, b=1.01$ [Kingni et al., 2014], and the initial condition $(0.7,2.3,-1.5)$, the system has only one stable equilibrium, whose characteristic values are $\lambda_{1}=-0.7679, \lambda_{2,3}=-0.0045 \pm 1.3012 i$. In this case, the system (1) has only one stable equilibrium, and no homoclinic (heteroclinic) orbits but has a single-scroll chaotic attractor (see Fig.2). The corresponding Lyapunov exponents [Wolf et al., 1985; Kuznetsov \& Leonov, 2005, 2007] are $L_{1}=0.094, L_{2}=0, L_{3}=-1.297$. The Kaplan-Yorke dimension [Kuznetsov et al., 2014] is defined by

$$
D_{L}=j+\frac{1}{\left|L_{j+1}\right|} \sum_{i=1}^{j} L_{i}
$$

where $j$ is the largest integer satisfying $\sum_{i=1}^{j} L_{i} \geq 0$ and $\sum_{i=1}^{j+1} L_{i}<0$. For the hyperchaotic attractor in Fig.5, the Kaplan-Yorke dimension is $D_{L}=2.072$, which means that the Lyapunov dimension of the hidden chaotic attractor is fractional. As cross section plot shown for $z=0$ with $a=1.3, b=1.01$ in Fig. 3 , a black dot is the stable equilibrium and surrounded by light blue. Moreover, the light blue region contains initial conditions $(\mathrm{x}, \mathrm{y}, 0)$ that attract to the stable equilibrium. The two black lines are a cross section of the strange attractor in the $z=0$ plane, and the red region contains initial conditions $(x, y, 0)$ that attract to the strange attractor.

In addition, the different dynamical regions in the space of the parameters $a$ and $b$ as determined in Fig.4. Initial conditions for each point in the plot are chosen randomly from a Gaussian distribution with unit variance centered on $(x, y, z)=(0,0,0)$. Thus regions of coexisting attractors are thus shown with colors intermingled, for example red and green in the narrow region around $(a, b)=(1.3,1.01)$ where a strange attractor coexists with a stable equilibrium. In particular, there is a narrow region around $(a, b)=(1.3,1.01)$ where chaotic solutions occur in the presence of a weakly stable focus equilibrium. In that region, the strange attractor is hidden. The region is small, and initial conditions must be chosen carefully. The basin plot shows the coexistence of the two attractors, which further indicates that the system has extremely rich dynamics Such systems belong to a newly introduced category of chaotic systems with hidden attractors that are important and potentially problematic in engineering applications.

\section{Infinity Dynamics in Kingni- Jafari system}

As we know, the Kingni- Jafari system (1) can be extended to an analytic system defined on a closed ball of radius one, whose interior is diffeomorphic to $\mathbb{R}^{3}$ and its invariant boundary, the 2- dimensional sphere $\mathbb{S}^{2}=\left\{(x, y, z) \mid x^{2}+y^{2}+z^{2}=1\right\}$ plays the role of the infinity. This ball shall be denoted here called the Poincaré ball, since the technique for doing this extension is the Poincaré compactification for a polynomial vector field in $\mathbb{R}^{n}$, which is described in detail in [Cima \& Llibre, 1990; Velasco, 1969]. 
Under the linear transformation $y \rightarrow y+\frac{b}{a}$, the only one equilibrium $E_{0}$ is transformed to the origin $O(0,0,0)$, and system (1) becomes

$$
\left\{\begin{array}{l}
\dot{x}=-z \\
\dot{y}=-x-z \\
\dot{z}=3 x-a y+x^{2}-z^{2}-y z-\frac{b}{a} z
\end{array}\right.
$$

In order to study the behavior of the trajectories of the system (4) near infinity, we will use the theory of Poincare compactification in $R^{3}$. Let Poincare ball $S^{3}=\left\{r=\left(r_{1}, r_{2}, r_{3}, r_{4}\right) \in R^{4} \mid\|r\|=1\right\}$ be the unit sphere, $S_{+}=\left\{r \in S^{3}, r_{4}>0\right\}$ and $S_{-}=\left\{r \in S^{3}, r_{4}<0\right\}$ be the northern and southern hemisphere, denote the tangent hyperplanes at the point $( \pm 1,0,0,0),(0, \pm 1,0,0),(0,0, \pm 1,0),(0,0,0, \pm 1)$ by the local chart $U_{i}, V_{i}$ for $i=1,2,3,4$, where $U_{i}=\left\{r \in S^{3}, r_{i}>0\right\}, V_{i}=\left\{r \in S^{3}, r_{i}<0\right\}$. We only consider the local chart $U_{i}, V_{i}$ for $i=1,2,3$ for getting the dynamics at $x, y, z$ infinity.

In the local charts $U_{1}$ and $V_{1}$

Take the change of variables $(x, y, z)=\left(w^{-1}, u w^{-1}, v w^{-1}\right)$, and $t=w \tau$, the system (4) becomes

$$
\left\{\begin{array}{l}
\frac{d u}{d \tau}=u v w-w-w v \\
\frac{d v}{d \tau}=w v^{2}+3 w-a w u+1-v^{2}-u v-\frac{b}{a} w v \\
\frac{d w}{d \tau}=v w^{2}
\end{array}\right.
$$

If $w=0$, the system (5) reduces to

$$
\left\{\begin{array}{l}
\frac{d u}{d \tau}=0 \\
\frac{d v}{d \tau}=1-v^{2}-u v
\end{array}\right.
$$

Solving the system (6) directly, one can obtain the solution

$$
\left\{\begin{array}{l}
u=c_{1}, \\
v=-\frac{c_{1}}{2}+\frac{1}{2} \tanh \left(\frac{t \sqrt{c_{1}^{2}+4}}{2}+\operatorname{arctanh} \frac{2 c_{2}+c_{1}}{\sqrt{c_{1}^{2}+4}}\right) \sqrt{c_{1}^{2}+4}
\end{array}\right.
$$

where $c_{1} \in R$. We find the general directions of the vector field in Fig.5. The flow in the local chart $V_{1}$ is the same as the flow in the local chart $U_{1}$ reversing the time, hence, the phase portrait on the infinite sphere at the negative end point of the $x$ axis is shown in Fig.5, reversing the time direction.

In the local charts $U_{2}$ and $V_{2}$

Next, we study the dynamics of the system (4) at infinity of the $y$ axis. Taking the transformation $(x, y, z)=\left(u w^{-1}, w^{-1}, v w^{-1}\right)$, and $t=w \tau$, the system (4) becomes

$$
\left\{\begin{array}{l}
\frac{d u}{d \tau}=w u^{2}+u v w-w v \\
\frac{d v}{d \tau}=u v w+w v^{2}+3 w u-w a+u^{2}-v^{2}-v-\frac{b}{a} w v \\
\frac{d w}{d \tau}=w^{2} u+w^{2} v
\end{array}\right.
$$

If $w=0$, the system (7) reduces to

$$
\left\{\begin{array}{l}
\frac{d u}{d \tau}=0 \\
\frac{d v}{d \tau}=u^{2}-v^{2}-v
\end{array}\right.
$$


Solving the system (8) directly, one can obtain the solution

$$
\left\{\begin{array}{l}
u=c_{1}, \\
v=-\frac{1}{2}+\frac{1}{2} \tanh \left(\frac{t \sqrt{4 c_{1}^{2}+1}}{2}+\operatorname{arctanh} \frac{2 c_{2}+c_{1}}{\sqrt{4 c_{1}^{2}+1}}\right) \sqrt{4 c_{1}^{2}+1},
\end{array}\right.
$$

where $c_{1} \in R$. We find the general directions of the vector field in Fig.6. The flow in the local chart $V_{2}$ is the same as the flow in the local chart $U_{2}$, hence, the phase portrait of the system (4) on the infinite sphere at the negative end point of the $y$ axis is shown in Fig.6, reversing the time direction.

In the local charts $U_{3}$ and $V_{3}$

Finally, we consider infinity at the $z$ axis. Let $(x, y, z)=\left(u w^{-1}, v w^{-1}, w^{-1}\right)$, and $t=w \tau$, the system (4) becomes

$$
\left\{\begin{array}{l}
\frac{d u}{d \tau}=-3 w u^{2}+a w u v-u^{3}+u+u v-w+\frac{b}{a} w u \\
\frac{d v}{d \tau}=-3 u v w+a w v^{2}-u^{2} v+v+v^{2}+\frac{b}{a} w v-w u-w \\
\frac{d w}{d \tau}=-3 u w^{2}+a w^{2} v-w u^{2}+w+w v+\frac{b}{a} w^{2}
\end{array}\right.
$$

If $w=0$, the system (9) reduces to

$$
\left\{\begin{array}{l}
\frac{d u}{d \tau}=-u^{3}+u+u v \\
\frac{d v}{d \tau}=-v u^{2}+v+v^{2}
\end{array}\right.
$$

The system (10) has an unstable node $(0,0)$ and a singular stable parabola $v=u^{2}-1$. Also, we can obtain the the local phase portraits as shown in Fig.7. The flow in the local chart $V_{3}$ is the same as the flow in the local chart $U_{3}$, hence, the phase portrait of the system (4) on the infinite sphere at the negative end point of the $z$ axis is shown in Fig.7, reversing the time direction. From above analysis, we have the following proposition. The phase portrait of the system (4) on the Poincare sphere at infinity is as shown in Fig.8. There exists two singular stable parabolas at the upper hemisphere and lower hemisphere and two nodes at the positive (unstable) and negative (stable) of the $z$ axis.

\section{Hopf bifurcation and generation of hidden chaos in the Kingni-Jafari system}

Suppose that the characteristic equation of Kingni-Jafari system (4) has a pair of pure imaginary roots $\pm i \omega\left(\omega \in R^{+}\right)$. It is easy to show that when $b=b_{0}=\frac{a^{2}}{3-a},(2)$ yields

$$
\lambda_{1}=\frac{a}{a-3}<0, \quad \lambda_{2,3}= \pm \sqrt{3-a} i
$$

where $0<a<3$.

Proposition 4.1. Define

$$
T=\left\{(a, b) \mid 0<a<3, b=b_{0}=\frac{a^{2}}{3-a},\right.
$$

then Jacobian matrix of system $(4)$ at $O(0,0,0)$ has one negative real eigenvalue $\frac{a}{a-3}$ and a pair of purely imaginary eigenvalues $\pm \sqrt{3-a} i$,

Taking $b$ as the Hopf bifurcation parameter, the transversally condition

$$
\left.\operatorname{Re}\left(\lambda^{\prime}\left(b_{0}\right)\right)\right|_{\lambda=\sqrt{3-a} i}=-\frac{(-3+a)^{3}}{2 a\left(-27+27 a-10 a^{2}+a^{3}\right)}<0
$$

is also satisfied. Therefore, we have the following theorem. 
Theorem 4.2. If $(a, b) \in T$ and $b$ varies and passes through the critical value $b_{0}=\frac{a^{2}}{3-a}$, system (4) undergoes Hopf bifurcation at the equilibrium $O(0,0,0)$.

We first review the projection method described in Chapters 3 and 5 [Kuznetsov 2004], but following the analysis of [Mello \& Coelho, 2009; Sotomayor et al., 2007a, 2007b], for the calculation of the first Lyapunov coefficient $l_{1}$, associated with the stability of a Hopf bifurcation. Consider the differential equation

$$
\dot{X}=f(X, \mu),
$$

where $X \in R^{3}$ and $\mu \in R^{2}$ are respectively vectors representing phase variables and control parameters. Assume that $f$ is a class of $C^{\infty}$ in $R^{3} \times R^{2}$. Suppose that (12) has an equilibrium point $X=X_{0}$ at $\mu=\mu_{0}$, and denoting the variable $X-X_{0}$ also by $X$, write

$$
F(X)=f\left(X, \mu_{0}\right)
$$

as

$$
F(X)=A X+\frac{1}{2} B(X, X)+\frac{1}{6} C(X, X, X)+O\left(\|X\|^{4}\right)
$$

where $A=f_{x}\left(0, \mu_{0}\right)$ and, for $i=1,2,3$,

$$
B(X, Y)=\left.\sum_{j, k=1}^{3} \frac{\partial^{2} F_{i}(\xi)}{\partial \xi_{j} \partial \xi_{k}}\right|_{\xi=0} X_{j} Y_{k}, C(X, Y, Z)=\left.\sum_{j, k, l=1}^{3} \frac{\partial^{3} F_{i}(\xi)}{\partial \xi_{j} \partial \xi_{k} \partial \xi_{l}}\right|_{\xi=0} X_{j} Y_{k} Z_{l} .
$$

Suppose that $A$ has a pair of complex eigenvalues on the imaginary axis: $\lambda_{2,3}= \pm i w_{0}\left(w_{0}>0\right)$, and these eigenvalues are the only eigenvalues with $\operatorname{Re} \lambda=0$. Let $T^{c}$ be the generalized eigenspace of $A$ corresponding to $\lambda_{2,3}$. Let $p, q \in C^{3}$ be vectors such that

$$
A q=i w_{0} q, A^{T} p=-i w_{0} p,\langle p, q\rangle=1,
$$

where $A^{T}$ is the transposed of the matrix $A$. Any vector $y \in T^{c}$ can be represented as $y=w q+\bar{w} \bar{q}$, where $w=\langle p, y\rangle \in C$. The two-dimensional center manifold associated to the eigenvalues $\lambda_{2,3}$ can be parameterized by $w$ and $\bar{w}$, by means of an immersion of the form $X=H(w, \bar{w})$, where $H: C^{2} \rightarrow R^{3}$ has a Taylor expansion of the form

$$
H(w, \bar{w})=w q+\bar{w} \bar{q}+\sum_{2 \leq j+k \leq 3} \frac{1}{j ! k !} h_{j k} w^{j} \bar{w}^{k}+O\left(|w|^{4}\right),
$$

with $h_{j k} \in C^{3}$ and $h_{j k}=h_{k j}^{-}$. Substituting this expression into (13) we obtain the following differential equation

$$
H_{w} w^{\prime}+H_{w} \bar{w}^{\prime}=F(H(w, \bar{w}))
$$

where $F$ is given by (13). The complex vectors $h_{i j}$ are obtained by solving the system of linear equations defined by the coefficients of (13). Taking into account the coefficients of $F$, system (13), on the chart $w$ for a central manifold, can be written as follows

$$
\dot{w}=i w_{0} w+\frac{1}{2} G_{21} w|w|^{2}+O\left(|w|^{4}\right)
$$

with $G_{21} \in C$. The first Lyapunov coefficient can be written as

$$
l_{1}=\frac{1}{2} \operatorname{Re} \mathrm{G}_{21},
$$

where $G_{21}=\left\langle p, C(q, q, \bar{q})+B\left(\bar{q}, h_{20}\right)+2 B\left(q, h_{11}\right)\right\rangle$. Defining $\mathcal{H}_{32}$ as

$$
\begin{aligned}
\mathcal{H}_{32}= & 6 B\left(h_{11}, h_{21}\right)+B\left(\bar{h}_{20}, h_{30}\right)+3 B\left(\bar{h}_{21}, h_{20}\right)+3 B\left(q, h_{22}\right) \\
& +2 B\left(\bar{q}, h_{31}\right)+6 C\left(q, h_{11}, h_{11}\right)+3 C\left(q, \bar{h}_{20}, h_{20}\right)+3 C\left(q, q, \bar{h}_{21}\right) \\
& +6 C\left(q, \bar{q}, h_{21}\right)+6 C\left(\bar{q}, h_{20}, h_{11}\right)+C\left(\bar{q}, \bar{q}, h_{30}\right) \\
& +D\left(q, q, q, \bar{h}_{20}\right)+6 D\left(q, q, \bar{q}, \bar{h}_{11}\right)+3 D\left(q, \bar{q}, \bar{q}, h_{20}\right) \\
& +E(q, q, q, \bar{q}, \bar{q})-6 G_{21} h_{21}-3 \bar{G}_{21} h_{21}
\end{aligned}
$$


and $G_{32}=\left\langle p, \mathcal{H}_{32}\right\rangle$, the second Lyapunov coefficient $l_{2}$ is given by

$$
l_{2}=\frac{1}{12} \operatorname{Re} \mathrm{G}_{32} .
$$

A Hopf bifurcation point $\left(X_{0}, \mu_{0}\right)$ is an equilibrium point of (12) where the Jacobian matrix $A$ only has a pair of purely imaginary eigenvalues $\pm i w_{0}\left(w_{0}>0\right)$, and the other eigenvalue with non-zero real part. At a Hopf point a two-dimensional center manifold is well defined, it is invariant under the flow generated by (12) and can be continued with arbitrary high class of differentiability to nearby parameter values.

A Hopf point is called transversal if the parameter-dependent complex eigenvalues cross the imaginary axis with non-zero derivative. In a neighborhood of a transversal Hopf point with $l_{1} \neq 0$ the dynamic behavior of the system (12), reduced to the family of parameter-dependent continuations of the center manifold, is orbitally topologically equivalent to the following complex normal form

$$
w^{\prime}=(\eta+i w) w+l_{1} w|w|^{2},
$$

where $w \in C, \eta, w$ and $l_{1}$ are real functions having derivatives of arbitrary higher order, which are continuations of $0, w_{0}$ and the first Lyapunov coefficient at the Hopf point [Sotomayor et al., 2007a]. As $l_{1}<0\left(l_{1}>0\right)$ one family of stable (unstable) periodic orbits can be found on this family of manifolds, shrinking to an equilibrium point at the Hopf point.

A Hopf point of codimension two is a Hopf point where $l_{1}$ vanishes. It is called transversal if $\eta=0$ and $l_{1}=0$ have transversal intersections, where $\eta=\eta(\mu)$ is the real part of the critical eigenvalues. In a neighborhood of a transversal Hopf point of codimension two with $l_{2} \neq 0$ the dynamic behavior of the system (12) reduced to the family of parameter-dependent continuations of the center manifold, is orbitally topologically equivalent to

$$
w^{\prime}=(\eta+i w) w+\tau w|w|^{2}+l_{2} w|w|^{4},
$$

where $\eta$ and $\tau$ are unfolding parameters.

In the rest of this section we employs the 3-dimensional Hopf bifurcation theory and applies symbolic computations to perform the analysis of parametric variations with respect to dynamical bifurcations. Because the system has only one equilibium, we consider the bifurcation of the system $(4)$ at $O(0,0,0)$ (system (1) at $E_{0}$ ).

Theorem 3.1. Consider the two-parameter family of differential equations (4). The first Lyapunov coefficient associated with the equilibrium $O$ is given by

$$
l_{1}=\frac{(a-3)\left(-648+1350 a-1185 a^{2}+551 a^{3}-131 a^{4}+12 a^{5}\right)}{a\left(2916-5832 a+4995 a^{2}-2295 a^{3}+586 a^{4}-77 a^{5}+4 a^{6}\right)} .
$$

If $l_{1}>0$ then the Hopf point at $O$ is unstable (weak repelling focus) and for each $b>b_{0}=\frac{a^{2}}{3-a}$, but close to $b_{0}$, there exists an unstable limit cycle near the asymptotically stable equilibrium $O$; if $l_{1}<0$ then the Hopf point at $O$ is asymptotically stable (weak attractor focus) and for each $b>b_{0}=\frac{a^{2}}{3-a}$, but close to $b_{0}$, there exists a stable limit cycle near the unstable equilibrium $O$.

Proof. Under this condition $0<a<3$, the transversality condition (11) is also satisfied. Accordingly, Hopf bifurcation at $O$ occurs. The stability of $O$ depends on the value of the first Lyapunov coefficient $l_{1}$, which shows the stability of the equilibrium point and the periodic orbits which appear. Using the notation of the previous section, the multilinear symmetric functions can be written as

$$
\begin{aligned}
B(x, y) & =\left(0,0,2 x_{1} y_{1}-2 x_{3} y_{3}-x_{2} y_{3}-x_{3} y_{2}\right), \\
C(x, y, z) & =(0,0,0,0) .
\end{aligned}
$$


Furthermore, we can also obtain

$$
\begin{aligned}
& p=\left(-\frac{a \sqrt{3-a}+3(a-3) i}{2\left[(3-a)^{3 / 2}+i a\right]},-\frac{i(3-a) a}{2\left[(3-a)^{3 / 2}+i a\right]}, \frac{(3-a)^{3 / 2}}{2\left[(3-a)^{3 / 2}+i a\right]}\right), \\
& q=\left(\frac{i}{\sqrt{3-a}}, \frac{-1+\sqrt{3-a} i}{3-a}, 1\right), \\
& h_{11}=\left(0,-\frac{2(1-a)}{(3-a) a}, 0\right), \\
& h_{20}=\left(-\frac{4(i+\sqrt{3-a})}{3\left[(3-a)^{3 / 2}-i a\right]}, \frac{10-4 a+6 \sqrt{3-a} i}{-6(a-3)^{2}+3 a \sqrt{3-a} i},-\frac{8(\sqrt{3-a}+(a-3) i)}{3\left[(3-a)^{3 / 2}-i a\right]}\right) .
\end{aligned}
$$

Then we can compute the following value

$$
G_{21}=\frac{-108+153 a-57 a^{2}+6 a^{3}+a\left(57-47 a+8 a^{2}\right) \sqrt{3-a} i}{3 a\left(-54+54 a-17 a^{2}+2 a^{3}\right)+9(3-a)^{3 / 2} a^{2} i}
$$

and

$$
l_{1}=\frac{(a-3)\left(-648+1350 a-1185 a^{2}+551 a^{3}-131 a^{4}+12 a^{5}\right)}{2 a\left(2916-5832 a+4995 a^{2}-2295 a^{3}+586 a^{4}-77 a^{5}+4 a^{6}\right)} .
$$

Therefore, the Theorem 3.1 is proved.

In order to investigate the relationship between the Hopf bifurcation and hidden chaos, we justify the above theoretical analysis of the first Lyapunov coefficient for the Hopf bifurcation of system (1) with $a=1.3$, according to Theorem 3.1, we have $b_{0} \doteq 0.9941$ and $l_{1} \doteq 0.0682>0$. Choosing initial condition $(0.1,0.87,-0.09)$, a unstable periodic solution should be found near the stable equilibrium point $E_{0}$ for $b=0.996>b_{0}$. This is indeed the case, as shown in Fig.8 (blue orbits). In addition, when parameters $a$ and $b$ keep unchanged, it shows that initial condition changes slightly and becomes $(0.15,0.87,-0.09)$, hidden chaotic attractor occurs from the unstable limit cycle that arose in the Hopf bifurcation (see Fig.8 (red orbits). Therefore, this system has a coexisting a point, a periodic cycle and a hidden chaotic attractor, demonstrating that this new system is truly complicated and interesting.

In the following theorem we study the sign of the second Lyapunov coefficient $l_{2}$ when the first coefficient $l_{1}$ vanishes.

Theorem 3.2. For system (1) there are two parameter sets

$$
D_{1}=\left\{(a, b) \mid a=a_{1}, b=\frac{a^{2}}{3-a}\right\}, D_{2}=\left\{(a, b) \mid a=a_{2}, b=\frac{a^{2}}{3-a}\right\},
$$

where $a_{1} \doteq 1.5359$ and $a_{2} \doteq 2.2768$ are the only two roots in the region $0<a<3$ for the $l_{1}=0$. The second Lyapunov coefficients at $E_{0}$ for parameter values $(a, b) \in D_{1}$ or $D_{2}$ are given by

$$
\left.l_{2}\right|_{D_{1}} \doteq-0.3423,\left.l_{2}\right|_{D_{2}} \doteq-0.2132 .
$$

Therefore, for the set of parameter values in $D_{1}$ or $D_{2}$, system (1) has a transversal Hopf point of codimension two at $E_{0}$ which is stable since $l_{2}<0$.

Proof. The second Lyapunov coefficient can be obtained for the parameters on the set $D_{1}$. For $a=a_{1}$, one has

$$
G_{21}=-0.37936 i
$$

and

$$
\begin{aligned}
& h_{21}=(-0.12824+0.01838 i,-0.35756-0.08761 i,-0.29128+0.15517 i), \\
& h_{30}=(0.65523+0.28148 i, 0.57769+0.46199 i, 1.02181-2.37852 i), \\
& h_{31}=(0.18321-0.09332 i, 0.22177-0.01762 i, 4.46976+0.87345 i), \\
& h_{22}=(0,0.20030,0), \\
& G_{32}=-4.10790-2.97868 i .
\end{aligned}
$$


By the above theorem and calculation, one has

$$
l_{1}=\frac{1}{2} \operatorname{Re} G_{21}=0,\left.\quad l_{2}\right|_{D}=\frac{1}{12} \operatorname{Re} G_{32}=-0.3423<0 .
$$

Similarly, we can get

$$
l_{1}=\frac{1}{2} \operatorname{Re} G_{21}=0,\left.\quad l_{2}\right|_{D}=\frac{1}{12} \operatorname{Re} G_{32}=-0.2132<0 .
$$

Therefore, we can arrive at the above Theorem 3.2.

\section{Zero-Hopf bifurcation and periodic orbits in Kingni- Jafari system}

We first present a result from the averaging theory [Sanders et al., 2007; Llibre \& Zhang, 2009; Llibre \& Valls, 2011; Llibre \& Chavela, 2014; Llibre et al., 2015].

Theorem 5.1. We consider the following two initial value problems

$$
\dot{x}=\varepsilon f(t, x)+\varepsilon^{2} g(t, x, \varepsilon), x(0)=x_{0},
$$

and

$$
\dot{y}=\varepsilon f^{0}(y), y(0)=y_{0},
$$

where $x, y, x_{0} \in \Omega$ an open subset of $R^{n}, t \in[0, \infty), \varepsilon \in\left(0, \varepsilon_{0}\right), f$ and $g$ are periodic of period $T$ in the variable $t$, and $f^{0}(y)$ is the averaged function of $f(t, x)$ with respect to $t$, i.e.,

$$
f^{0}(y)=\frac{1}{T} \int_{0}^{T} f(t, y) d t
$$

Suppose:

(i) $f$, its Jacobian $\frac{\partial f}{\partial x}$, its Hessian $\frac{\partial^{2} f}{\partial x^{2}}, g$, its Jacobian $\frac{\partial g}{\partial x}$ are defined, continuous and bounded by a constant independent on $\varepsilon \in[0, \infty) \times \Omega$ and $\varepsilon \in\left(0, \varepsilon_{0}\right)$;

(ii) $T$ is a constant independent of $\varepsilon$;

(iii) $y(t)$ belongs to on the interval of time $[0,1 / \varepsilon]$. Then the following statements hold [8, 10]:

(a) For the time scale $1 / \varepsilon$, we have that $x(t)-y(t)=O(\varepsilon)$, as $\varepsilon \rightarrow \rightarrow 0$.

(b) If $p$ is a singular point of the averaged system (16) such that the determinant of the Jacobian matrix is not zero, then there exists a limit cycle $\phi(t, \varepsilon)$ of period $T$ for system (15) which is close to $p$ and such that $\phi(t, \varepsilon) \rightarrow p$ as $\varepsilon \rightarrow 0$.

(c) The stability or instability of the limit cycle $\phi(t, \varepsilon)$ is given by the stability or instability of the singular point $p$ of the averaged system (16). In fact, the singular point $p$ has the stability behaviour of the Poincaré map associated to the limit cycle $\phi(t, \varepsilon)$.

It is obvious that the averaging method gives a quantitative relation between the solutions of some non-autonomous periodic differential system and the solutions of its averaged differential system, which is an autonomous one. It provides a first order approximation in $\varepsilon$ for the limit cycles of a periodic differential system.

We recall that an equilibrium point is a zero-Hopf equilibrium of a 3-dimensional autonomous differential system, if it has a zero real eigenvalue and a pair of purely imaginary eigenvalues. Therefore, this system (1) has no zero-Hopf bifurcations from Eq.(2). In order to exhibit different topological type of dynamics in the small neighborhood of this isolated equilibrium as the two parameters vary in a small neighborhood of the origin. In the next result, we characterize the equilibrium point $(0,0,0)$ is zero-Hopf equilibrium when Kingni- Jafari system (1) has the parameter $b=k a^{2}$ :

$$
\left\{\begin{array}{l}
\dot{x}=-z \\
\dot{y}=-x-z \\
\dot{z}=3 x-a y+x^{2}-z^{2}-y z-k a z
\end{array}\right.
$$


According to the condition (3), the equilibrium point $(0,0,0)$ is stable when $k>\frac{1}{3-a}$. Therefore, we limited $k>\frac{1}{3-a}$.

Proposition 5.2. The differential system (18) has a unique zero-Hopf equilibrium localized at the origin of coordinates when $a=0$. Moreover, the eigenvalues at the origin for this one-parameter family are 0 , and $\pm \sqrt{3} i$.

We shall study when the system has a zero-Hopf bifurcation producing some unstable periodic orbit for $a>0$ sufficiently small that bifurcate from a zero-Hopf equilibrium point localized at the origin of coordinates when $a=0$. It is worth noting that unstable periodic orbit has been numerically extracted from chaotic attractors of some continuous dynamical systems. By those unstable periodic solutions it has been attempted to explain typical behavior and statistical properties of the chaotic solutions. Therefore, we have the following result.

Theorem 5.3. When $k>\frac{1}{3-a}$, the system (18) has a zero-Hopf bifurcation at the equilibrium point $(0,0,0)$ when $a=0$, and produce one small periodic solution for $a>0$ sufficiently small:

$x=\frac{a \sqrt{2(3 k-1)} \cos \theta}{\sqrt{3}}, y=\frac{a \sqrt{2(3 k-1)} \cos \theta}{\sqrt{3}}-\frac{a \sqrt{2(3 k-1)} \sin \theta}{3}+\frac{a(1-3 k)}{3}, z=a \sqrt{2(3 k-1)} \sin \theta$.

Moreover, the periodic solution is unstable and has two invariant manifolds, one stable and one unstable, each of them formed by two cylinders.

Proof. In order to find the changes of variables for writing our differential system (18) in the normal form for applying the averaging theory, first we write the linear part at the origin of the differential system (18) when $a=0$ into its real Jordan normal form, i.e. into the form

$$
\left(\begin{array}{ccc}
0 & \sqrt{3} & 0 \\
-\sqrt{3} & 0 & 0 \\
0 & 0 & 0
\end{array}\right)
$$

For doing that we consider the linear change

$$
x=\frac{u}{\sqrt{3}}, y=\frac{u}{\sqrt{3}}-\frac{v}{3}+w, z=v .
$$

Using the new variables $(u, v, w)$, the differential system (18) can be written as

$$
\left\{\begin{array}{l}
\dot{u}=-\sqrt{3} v \\
\dot{v}=\left(\sqrt{3}-\frac{1}{\sqrt{3}} a\right) u+\left(\frac{1}{3} a-a k\right) v-a w+\frac{u^{2}}{3}-\frac{2 v^{2}}{3}-\frac{u v}{\sqrt{3}}-v w, \\
\dot{w}=-\frac{a}{3 \sqrt{3}} u+\left(\frac{a}{9}-\frac{a k}{3}\right) v-\frac{a w}{3}+\frac{u^{2}}{9}-\frac{u v}{3 \sqrt{3}}-\frac{2 v^{2}}{9}-\frac{v w}{3} .
\end{array}\right.
$$

Now we write the differential system (19) in cylindrical coordinates $(u, v)=(r \cos \theta, r \sin \theta)$

$$
\left\{\begin{array}{l}
\dot{r}=-\frac{1}{6} \sin \theta\left(r^{2}+6 a w+2 \sqrt{3} a r \cos \theta-3 r^{2} \cos 2 \theta-2 a r \sin \theta+6 a k r \sin \theta+6 r w \sin \theta+\sqrt{3} r^{2} \sin 2 \theta\right), \\
\dot{\theta}=\sqrt{3}-\frac{1}{6} r \cos \theta-\frac{a w \cos \theta}{r}-\frac{a \cos ^{2} \theta}{\sqrt{3}}+\frac{1}{2} r \cos \theta \cos 2 \theta+\left(\frac{1}{3} a-a k-w\right) \cos \theta \sin \theta-\frac{r \cos \theta \sin 2 \theta}{2 \sqrt{3}}, \\
\dot{w}=\frac{1}{18}\left(-r^{2}-6 a w-2 \sqrt{3} a r \cos \theta+3 r^{2} \cos 2 \theta+2 a r \sin \theta-6 a k r \sin \theta-6 r w \sin \theta-\sqrt{3} r^{2} \sin 2 \theta\right) .
\end{array}\right.
$$

If the rescaling $(r, w)=(\varepsilon R, \varepsilon W)$ with $a=\varepsilon>0$ a sufficiently small parameter, then the system (20) becomes

$$
\left\{\begin{array}{l}
\frac{d R}{d \theta}=\varepsilon F_{11}(\theta, R, W)+O\left(\varepsilon^{2}\right), \\
\frac{d W}{d \theta}=\varepsilon F_{21}(\theta, R, W)+O\left(\varepsilon^{2}\right),
\end{array}\right.
$$


where

$$
\begin{aligned}
F_{11}(\theta, R, W)= & -\frac{\sin \theta}{6 \sqrt{3}}\left(R^{2}+6 W+2 \sqrt{3} R \cos \theta-3 R^{2} \cos 2 \theta-2 R \sin \theta+6 k R \sin \theta\right. \\
& \left.+6 R W \sin \theta+\sqrt{3} R^{2} \sin 2 \theta\right), \\
F_{21}(\theta, R, W)= & -\frac{1}{18 \sqrt{3}}\left(R^{2}+6 W+2 \sqrt{3} R \cos \theta-3 R^{2} \cos 2 \theta-2 R \sin \theta+6 k R \sin \theta\right. \\
& \left.+6 R W \sin \theta+\sqrt{3} R^{2} \sin 2 \theta\right) .
\end{aligned}
$$

Using the notation of the averaging theory we have that if we take $t=\theta, T=2 \pi, x=(R, W)$. It is immediate to check that system (21) satisfies all the assumptions of the averaging theory for periodic orbits.

Now we denote $g(R, W)=\left(g_{11}(R, W), g_{21}(R, W)\right)$ and compute the integrals $(21)$, i.e. the averaged functions

$$
\left\{\begin{array}{l}
g_{11}(R, W)=\frac{1}{2 \pi} \int_{0}^{2 \pi} F_{11}(\theta, R, W) d \theta=-\frac{R(-1+3 k+3 W)}{6 \sqrt{3}} \\
g_{21}(R, W)=\frac{1}{2 \pi} \int_{0}^{2 \pi} F_{21}(\theta, R, W) d \theta=-\frac{R^{2}+6 w}{18 \sqrt{3}}
\end{array}\right.
$$

Therefore, the $g_{11}(\theta, R, W)=g_{21}(\theta, R, W)=0$ has an unique positive real solution $\left(R_{*}, W_{*}\right)$ when $k>\frac{1}{3}$, where

$$
R_{*}=\sqrt{2(3 k-1)}, W_{*}=\frac{1}{3}(1-3 k) .
$$

In addition, we know the Jacobian at the point $\left(R_{*}, W_{*}\right)$ is

$$
\frac{1-3 k}{27}<0
$$

and the eigenvalues at the solution $\left(R_{*}, W_{*}\right)$ are

$$
\frac{-\sqrt{3} \pm 3 \sqrt{-1+4 k}}{18} .
$$

This shows that periodic orbit is unstable having a stable manifold and an unstable manifold both formed by two cylinders. Therefore, it guarantees for $\varepsilon>0$ sufficiently small the existence of a periodic solution $(R, W) \rightarrow\left(R_{*}, W_{*}\right)$ when $\varepsilon \rightarrow 0$. So system (18) for $\varepsilon \neq 0$ sufficiently small has one periodic orbit $\chi_{\varepsilon} \rightarrow\left\{w=W_{*}\right\} \bigcap\left\{u^{2}+v^{2}=R_{*}^{2}\right\}$. Going back to the differential system (20), we get that such a system for $a=\varepsilon>0$ sufficiently small has one periodic solution of period approximately $2 \pi$ of the form

$$
(r, w)=\left(\varepsilon R_{*}+O(a), \varepsilon W_{*}+O(a)\right) .
$$

Moreover, the differential system (19) into two periodic solutions of period also close to $2 \pi$ of the form

$$
u(t)=a \sqrt{2(3 k-1)} \cos \theta, v(t)=a \sqrt{2(3 k-1)} \sin \theta, w(t)=\frac{a(1-3 k)}{3} .
$$

for $a>0$ sufficiently small. Finally, we get for the differential system (18) the the periodic solution

$$
x=\frac{a \sqrt{2(3 k-1)} \cos \theta}{\sqrt{3}}, y=\frac{a \sqrt{2(3 k-1)}(\sqrt{3} \cos \theta-\sin \theta)}{3}+a(1-3 k), z=a \sqrt{2(3 k-1)} \sin \theta,
$$

$a>0$ is sufficiently small. It is a periodic solution starting at the zero-Hopf equilibrium point. This completes the proof of Theorem 5.3.

For $a=0.0001, k=10$ and initial condition $(4.3970 a,-5.2697 a, 0)$, we obtain the numerical phase diagram (the red parts in Fig.9). In addition, according to the solution (22), we also give the theoretical orbit from averaging method (the black parts in Fig.9). It is easy to find the correctness and effectiveness of the analytical results. In some cases the zero-Hopf bifurcation could imply a local birth of "chaos", see for instance the articles [Scheurle \& Marsden 1984; Champneys \& Kirk 2004; Baldomá \& Seara 2006]. 


\section{Conclusion}

It is apparent that the Kingni-Jafari system belongs to the newly introduced class of chaotic systems with hidden attractors. There are still abundant and complex dynamical behaviors and the topological structure of the new system should be completely and thoroughly investigated and exploited. In this paper, some complex dynamics of the Kingni-Jafari system have been studied theoretically. A complete description of the dynamics of this system at infinity is given using the Poincaré compactification of polynomial vector in $\mathbb{R}^{3}$. The generation mechanism of the hidden chaos for this special system is investigated. It exhibits the result of hidden chaos from a Hopf bifurcation point. In order to determinate the Lyapunov stability when the first Lyapunov coefficient vanishes, we make an extension of the analysis to degenerate cases. In addition, in the certain parameter region that the equilibrium is stable, an analytic proof of the existence of zero-Hopf bifurcation is exhibited by the averaging theory. A unstable periodic orbit from the zero- Hopf bifurcation are obtained. This approach may be useful to clarify the complicated phenomena that hidden chaotic attractors coexist with stable equilibrium.

Indeed, the global dynamical behaviors and the geometrical structure of this system have not been presented completely. It is expected that more detailed theory analysis and simulation investigations will be provided in a forthcoming study.

\section{Acknowledgements}

We would like to express our gratitude to the referees for their valuable comments and suggestions that led to a truly significant improvement of the manuscript. This work was supported by the Polish National Science Centre, MAESTRO Programme-Project (No. 2013/08/A/ST8/00/780), the Natural Science Foundation of China (No. 11401543), the China Scholarship Council (CSC) (No. 201506415023), Beijing Postdoctoral Research Foundation (No. 2015ZZ17), the China Postdoctoral Science Foundation funded project (No. 2014M560028 and No.2015T80029), the Fundamental Research Funds for the Central Universities, China University of Geosciences (Wuhan) (No. CUGL150419), the Government of Chaoyang District Postdoctoral Research Foundation (No. 2015ZZ-7) and the Funding Project for Academic Human Resources Development in Institutions of Higher Learning under the Jurisdiction of Beijing Municipality (PHRIHLB).

\section{References}

Baldomá, I., Seara, T. M. [2006] "Breakdown of heteroclinic orbits for some analytic unfoldings of the Hopf-Zero singularity," J. Nonlinear Sci. 16, 543-582.

Champneys, A. R., Kirk, V. [2004] "The entwined wiggling of homoclinic curves emerging from saddlenode/Hopf instabilities," Physica D 195, 77-105.

Cima, A., Llibre, J. [1990] "Bounded polynomial vector fields," Trans. Am. Math. Soc. 318, 557-579.

Dudkowski, D., Prasad, A. \& Kapitaniak, T. [2015] "Perpetual points and hidden attractors in dynamical systems," Phys. Lett. A 379, 2591-2596.

Jafari, S., Sprott, J. C. [2013] "Simple chaotic flows with a line equilibrium," Chaos Soliton. Fract. 57, 79-84.

Jafari, S., Sprott, J. C. \& Golpayegani, S. M. R. H. [2013] "Elementary quadratic chaotic flows with no equilibria," Phys. Lett. A 377, 699-702.

Jafari, S., Sprott, J. C. \& Nazarimehr F. [2015] " Recent New Examples of Hidden Attractors," Eur. Phys. J. Special Topics 224, 1469-1476.

Kapitaniak, T., Leonov, G. A. [2015] "Multistability: Uncovering hidden attractors," Eur. Phys. J. Special Topic 224, 1405-1408.

Kingni, S. T., Jafari, S., Simo, H. \& Woafo, P. [2014] "Three dimensional chaotic autonomous system with only one stable equilibrium: analysis, circuit design, parameter estimation, control, synchronization and its fractional-order form," Eur. Phys. J. Plus 129, 1-16. 
Kuznetsov, N. V., Alexeeva, T. A. \& Leonov, G. A. [2014] "Invariance of Lyapunov characteristic exponents, Lyapunov exponents, and Lyapunov dimension for regular and non-regular linearizations," arXiv:1410.2016v2, 2014 [http://arxiv.org/pdf/1410.2016v2.pdf].

Kuznetsov, N. V., Leonov, G. A. [2005] "On stability by the first approximation for discrete systems," 2005 International Conference on Physics and Control 1514053, 596-599.

Kuznetsov, N. V., Leonov, G. A. [2007] "Time-varying linearization and the Perron effects," Internat. J. Bifur. Chaos 17, 1079-1107.

Kuznetsov, N. V., Leonov, G. A. \& Seledzhi, S. M. [2011a] "Hidden oscillations in nonlinear control systems," IFAC Proc. Vol. (IFAC-PapersOnline) 18, 2506-2510.

Kuznetsov, N. V., Kuznetsova, O. A., Leonov, G. A. \& Vagaytsev, V. I. [2011b] "Hidden attractor in Chua's circuits," ICINCO 2011 - Proc. 8th Int. Conf. Informatics in Control, Automation and Robotics, $27-283$.

Kuznetsov, Y. A. [2004] "Elements of Applied Bifurcation Theory," New York, Springer-Verlag.

Lao, S. K., Shekofteh. Y., Jafari, S. \& Sprott, J. C. [2014] "Cost function based on gaussian mixture model for parameter estimation of a chaotic circuit with a hidden attractor," Internat. J. Bifur. Chaos 24, 1450010.

Leonov, G. A., Kuznetsov, N. V. \& Vagaitsev, V. I. [2011] "Localization of hidden Chua's attractors," Phys. Lett. A 375, 2230-2233.

Leonov, G. A., Kuznetsov, N. V. \& Vagaitsev, V. I. [2012] "Hidden attractor in smooth Chua systems," Physica D 241, 1482-1486.

Leonov, G. A., Kuznetsov, N. V. [2013] "Hidden attractors in dynamical systems. From hidden oscillations in Hilbert-Kolmogorov, Aizerman, and Kalman problems to hidden chaotic attractor in Chua circuits," Internat. J. Bifur. Chaos 23, 1330002.

Leonov, G. A., Kuznetsov, N. V., Kiseleva, M. A., Solovyeva, E. P. \& Zaretskiy, A. M. [2014] "Hidden oscillations in mathematical model of drilling system actuated by induction motor with a wound rotor," Nonlinear Dyn. 77, 277-288.

Li, C. B., Sprott, J. C. [2014a] "Finding coexisting attractors using amplitude control," Nonlinear Dyn. 78, 2059-2064.

Li, C. B., Sprott, J. C. [2014b] "Coexisting hidden attractors in a 4-D simplified Lorenz system," Internat. J. Bifur. Chaos 24, 1450034.

Llibre, J., Chavela, E. P. [2014] "Zero-Hopf bifurcation for a class of Lorenz-type systems," Discrete Continuous Dyn. Syst. Ser. B 19, 1731-1736.

Llibre, J., Regilene, D. S. O.\& Claudia, V. [2015] "On the integrability and the zero-Hopf bifurcation of a Chen-Wang differential system," Nonlinear Dyn. 80, 353-361.

Llibre, J., Valls, C. [2011] "Hopf bifurcation for some analytic differential systems in $\mathbb{R}^{3}$ via averaging theory," Discrete Continuous Dyn. Syst. Ser. B 30, 779-790.

Llibre, J., Zhang, X. [2009] "Hopf bifurcation in higher dimensional differential systems via the averaging method," Pacific J. Math. 240, 321-341.

Mello, L. F., Coelho,S. F. [2009] "Degenerate Hopf bifurcations in the Lü system," Phys. Lett. A 373, $1116-1120$.

Molaie, M., Jafari, S., Sprott, J. C. \& Golpayegani, S. M. R. H. [2013] "Simple chaotic flows with one stable equilibrium," Internat. J. Bifur. Chaos 23, 1350188.

Sanders, J.A., Verhulst, F. \& Murdock, J. [2007] Averaging methods in nonlinear dynamical systems. In: Applied Mathematical Sciences. 2nd ed., vol. 59. Springer, New York.

Scheurle, J., Marsden, J. [1984] "Bifurcation to quasi-periodic tori in the interaction of steady state and Hopf bifurcations," SIAM J. Math. Anal. 15, 1055-1074.

Shahzad, M., Pham, V. T., Ahmad, M. A., Jafari, S., \& Hadaeghi, F. [2015] "Synchronization and circuit design of a chaotic system with coexisting hidden attractors," Eur. Phys. J. Special Topics 224, 16371652.

Sharma, P. R., Shrimali, M. D., Prasad, A., Kuznetsov, N. V. \& Leonov, G. A. [2015a] "Control of multistability in hidden attractors," Eur. Phys. J. Special Topics 224, 1485-1491.

Sharma, P. R., Shrimali, M. D., Prasad, A., Kuznetsov, N. V. \& Leonov, G. A. [2015b] "Controlling 
dynamics of hidden attractors," Internat. J. Bifur. Chaos 25, 1550061.

Silva, C. P. [1993] "Shil'nikov's theorem-a tutorial," IEEE Trans. Circ. Syst.-I: Fund. Th. Appl. 40, 657682.

Sotomayor, S., Mello, L. F. \& Braga, D. C. [2007a] "Bifurcation analysis of the Watt governor system," Comm. Appl. Math. 26, 19-44.

Sotomayor, S., Mello, L. F. \& Braga, D. C. [2007b] "Lyapunov coefficients for degenerate Hopf bifurcations," arXiv:0709.3949v1 [math.DS], http://arxiv.org/.

Sprott, J. C., Wang, X. \& Chen, G. R. [2013] "Coexistence of point, periodic and strange attractors," Int. J. Bifurcation and Chaos 23, 1350093.

Velasco, E. A. G. [1969] "Generic properties of polynomial vector fields at infinity," Trans. Am. Math. Soc. 143, 201-221.

Wang, X. \& Chen, G. R. [2012] "A chaotic system with only one stable equilibrium," Commun. Nonlin. Sci. Numer. Simulat. 17, 1264-1272.

Wang, Z., Cang, S., Ochola, E. O. \& Sun, Y. [2012] "A hyperchaotic system without equilibrium," Nonlin. Dyn. 69, 531-537.

Wang, Z., Sun, W., Wei, Z. C. \& Zhang, S. W. [2015] "Dynamics and delayed feedback control for a 3D jerk system with hidden attractor," Nonlin. Dyn. 82, 577-588.

Wei, Z. C. [2011] "Dynamical behaviors of a chaotic system with no equilibria," Phys. Lett. A 376, 102-108.

Wei, Z. C., Wang, R. \& Liu A. [2014] "A new finding of the existence of hidden hyperchaotic attractors with no equilibria," Math. Comput. Simulat. 100, 13-23.

Wei, Z. C., Yang, Q. G. [2011] "Dynamical analysis of a new autonomous 3-D chaotic system only with stable equilibria," Nonlin. Anal.: Real World Appl. 12, 106-118.

Wei, Z. C., Yang, Q. G. [2012] "Dynamical analysis of the generalized Sprott C system with only two stable equilibria," Nonlinear Dyn. 68, 543-554.

Wei, Z. C., Zhang, W. [2014] "Hidden hyperchaotic attractors in a modified Lorenz-Stenflo system with only one stable equilibrium," Internat. J. Bifur. Chaos 24, 1450127.

Wolf, A., Swift, J. B., Swinney, H. L. \& Vastano, J. A. [1985] "Determining Lyapunov exponents from a time series," Physica D 16, 285-317.

Yang, Q. G., Wei, Z. C. \& Chen, G. R. [2010] "An unusual 3D autonomous quadratic chaotic system with two stable node-foci," Internat. J. Bifur. Chaos 20, 1061-1083. 


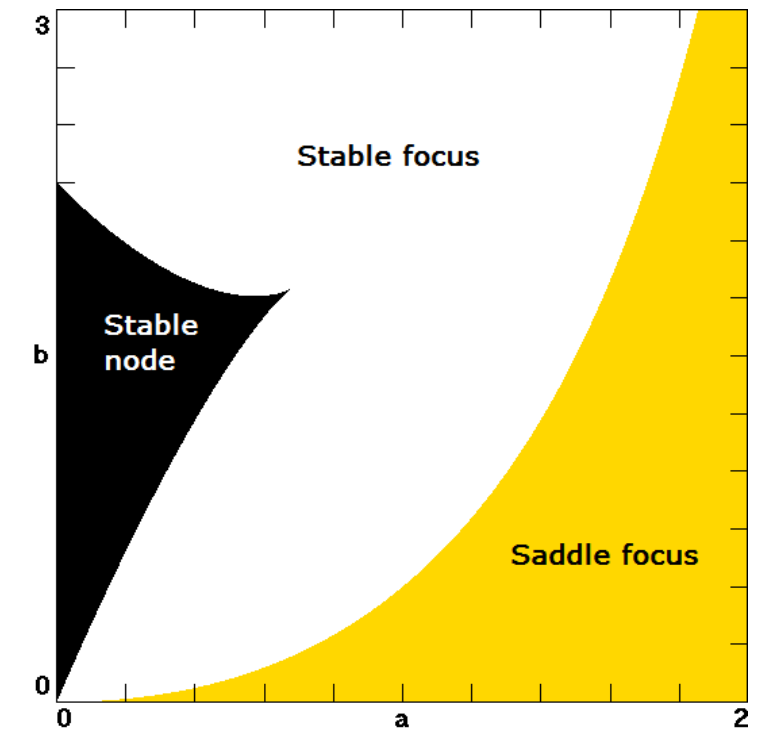

Fig.1. The equilibrium point $E_{0}$ of system (1) is stable node in the black region, stable focus in white region and saddle focus in yellow region. 

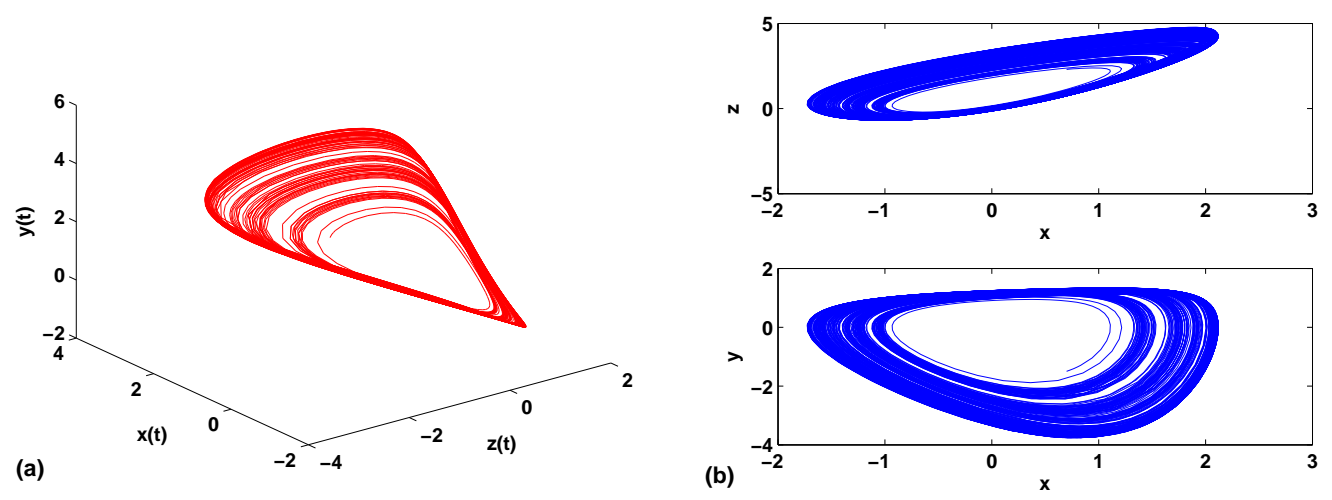

Fig.2. Hidden chaotic attractors of system (1) with parameters $a=1.3, b=1.01$ and initial condition $(0.7,2.3,-1.5)$.

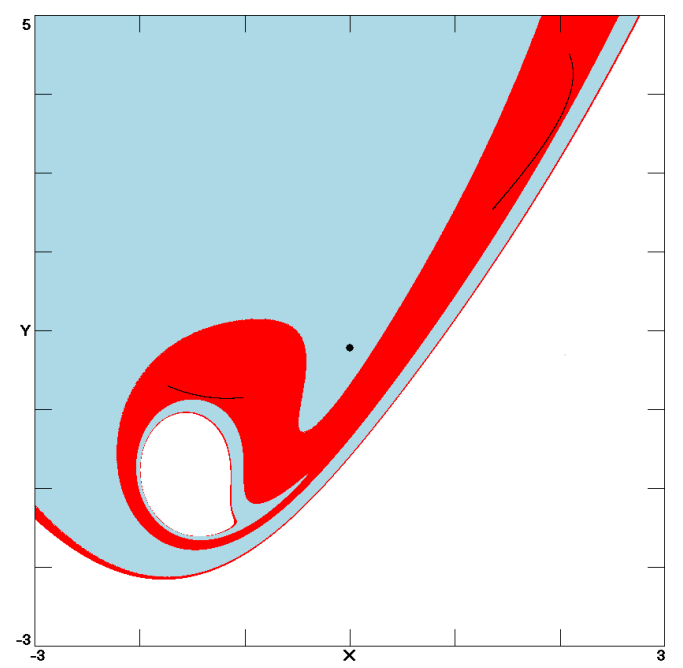

Fig.3. For system (1), the strange attractor basin in red and the stable equilibrium basin in light blue for $z=0$ with $a=1.3, b=1.01$. 


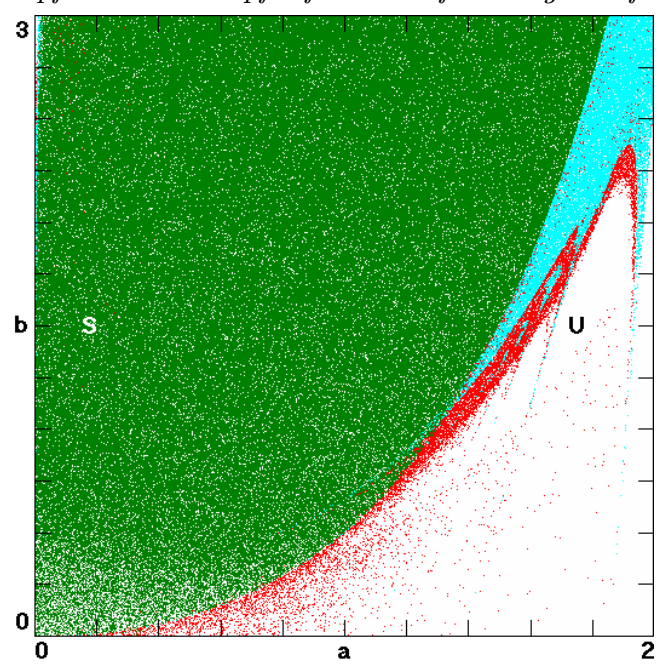

Fig.4. Regions of various dynamical behaviors as a function of the bifurcation parameters $a$ and $b$. The region for stable equilibrium are shown in green, the chaotic regions are shown in red, the limit cycles are shown in light Blue, and unbounded solutions are shown in white.

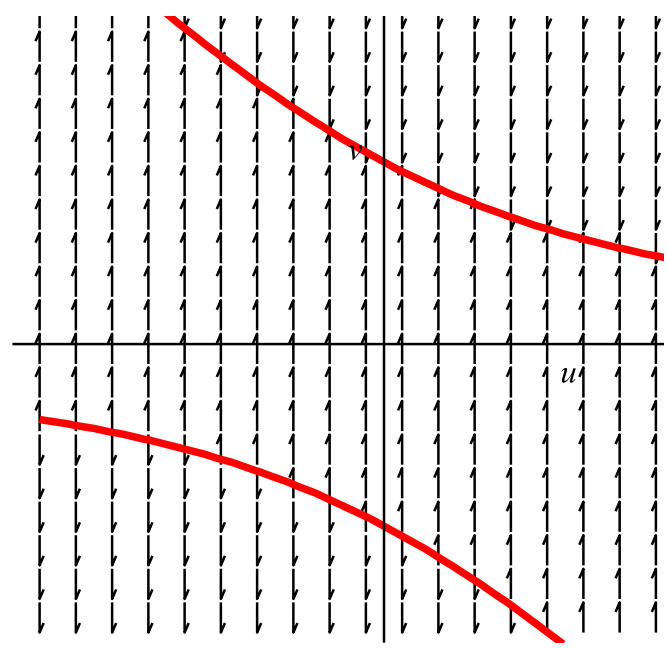

Fig.5. Trajectories in the local phase portraits of system (6). 


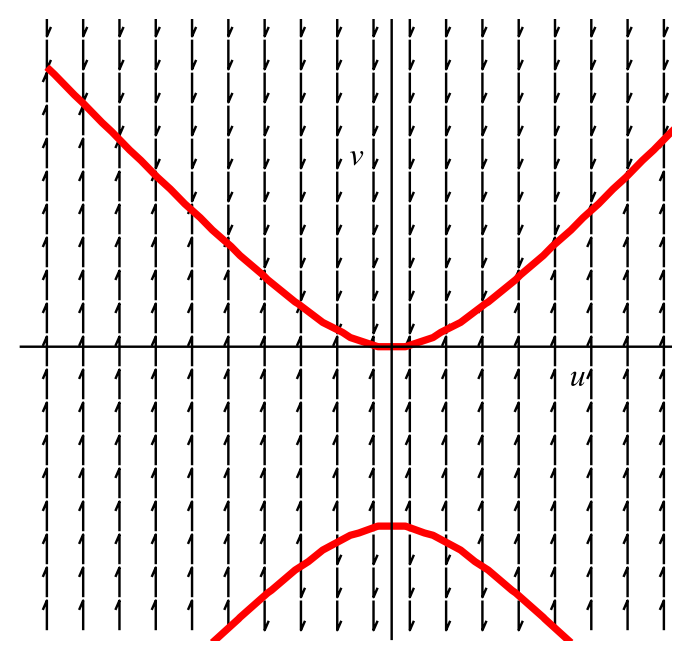

Fig.6. Trajectories in the local phase portraits of system (8).

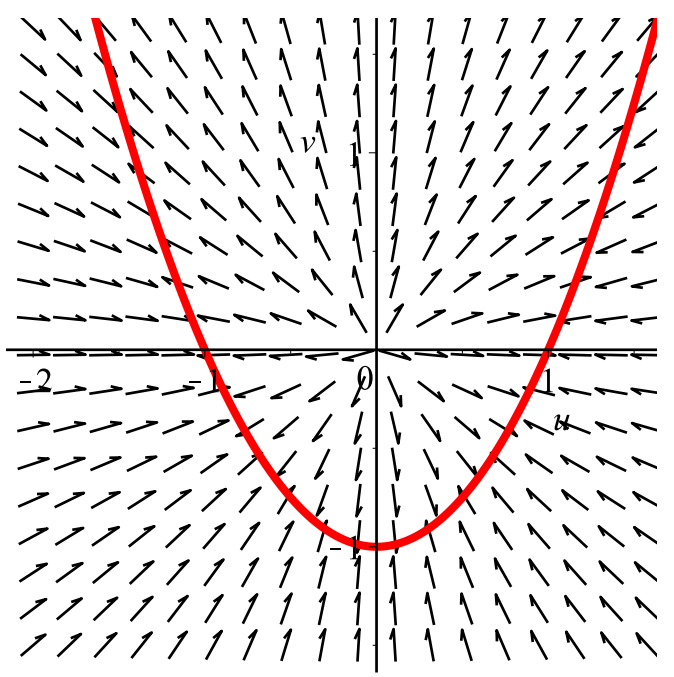

Fig.7. Trajectories in the local phase portraits of system (10). 


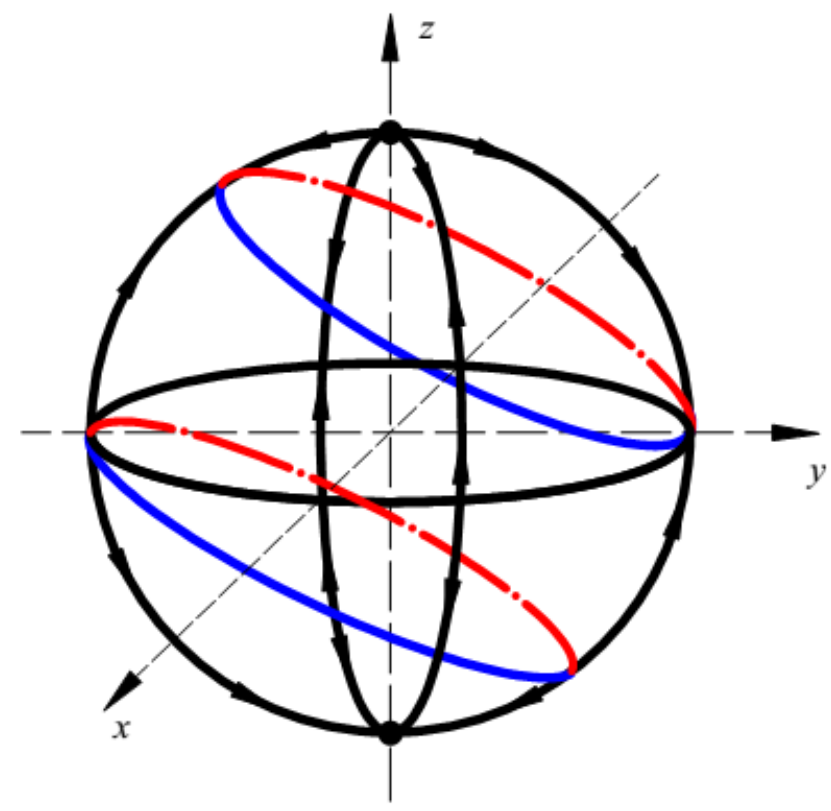

Fig.8. Phase portrait of the system (4) on the Poincare sphere at infinity.
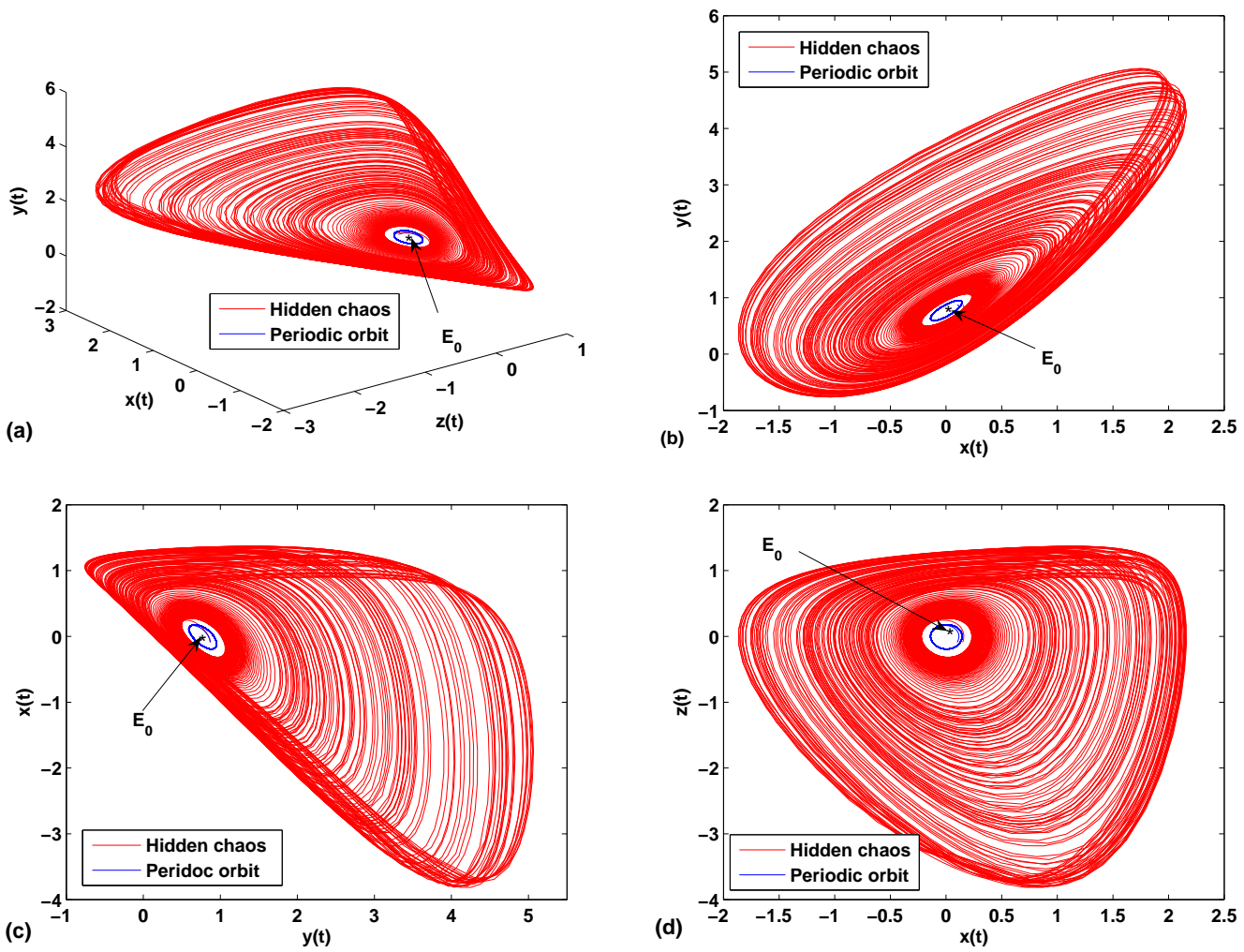

Fig.9. Coexistence of hidden chaotic attractor and periodic orbit of system (1) with parameters $a=1.3, b=0.996>b_{0} \doteq 0.9941$; the hidden chaotic attractor (red) from initial condition $(0.15,0.87,-0.09)$ and the periodic attractor (blue) from initial condition $(0.1,0.87,-0.09)$. 

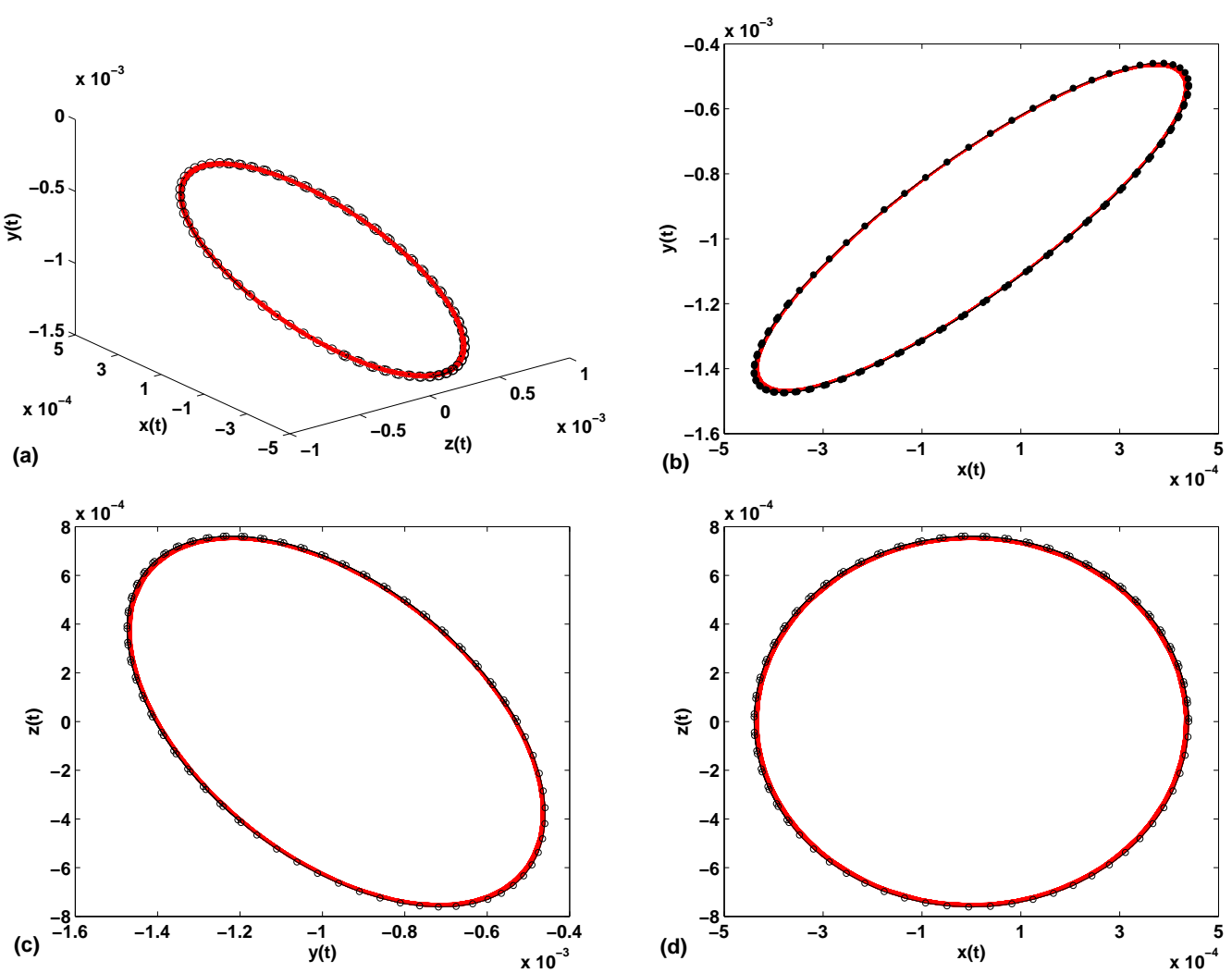

Fig.10. Phase diagram (red parts) and theoretical approximate orbit (black parts) of system (18) with $a=0.0001, k=10$ and initial condition $(4.3970 a,-5.2697 a, 0)$. 